\title{
Nonlinear sampled-data stabilization with delays
}

Salvatore Monaco, Dorothée Normand-Cyrot and Mattia Mattioni

\begin{abstract}
In this work, how sampling can be instrumental for stabilizing nonlinear dynamics with delays is discussed through several approaches developed by the authors in a comparative perspective with respect to the existing literature. Performances and computational aspects are illustrated through academic examples.
\end{abstract}

\section{Introduction}

Several recent approaches (see among them $[1,2,3,4,5,6]$ ) discuss the compensation of delays for nonlinear continuous-time systems in terms of reduction, descriptor or predictor-based strategies. Simultaneously, mainly motivated by implementation issues, a growing interest has been addressed toward systems under sampling. In particular, robustness of sample-and-hold stabilizing controllers with respect to actuators uncertainties (see [7] and the references therein) or with respect to delays are investigated in the literature $[8,9,10,11,12,13,14,15]$. These contributions are essentially concerned with implementation of existing feedback laws designed over the continuos plant or a priori assuming the existence of ad-hoc sampled-data controllers for the delay free model. That sampling is instrumental when dealing with time delays is even more clear when considering nonlinear dynamics affected

S. Monaco

Dipartimento di Ingegneria Informatica, Automatica e Gestionale, DIAG Università di Roma "La Sapienza", via Ariosto 25, 00185 Roma, Italy, e-mail: salvatore.monaco@uniroma1.it

D. Normand-Cyrot

Laboratoire des Signaux et Systèmes (L2S, UMR CNRS 8506) CNRS; 3, rue Joliot Curie, 91192 ,

Gif-sur-Yvette, France e-mail: cyrot@12s.centralesupelec.fr

M. Mattioni

L2S (CNRS and Université Paris-Sud) and DIAG (Università di Roma 'La Sapienza') with mobility support from the Université Franco-Italienne/Università Italo-Francese (UFI/UIF), e-mail: mattia.mattioni@12s.centralesupelec.fr 
by fixed and known input or measurements delays. In this case, sampled-data predictor based schemes (see $[10,12])$ can be exactly computed for particular classes of systems admitting closed form (possibly finitely computable) expressions of the predictor map.

The purpose of this work is to go further in the direct design of sampled-data feedback laws for input-delayed dynamics and investigate some simple extensions to special classes of systems affected by state delays. The results stated by the authors for the class of input-affine dynamics in some recent works [16, 17, 18, 19] are in the sequel extended to more general classes of nonlinear time-delayed systems with several comments on some open perspectives.

Throughout the paper we address the problem of designing sampled-data controllers of a given continuous input-delayed dynamics starting from its sampled equivalent model; the resulting control laws admit parametrized expressions in the sampling period which can be used to underline the possible advantage of sampling. The design is developed in three steps starting by showing how to get global asymptotic stabilization of the equivalent sampled delay free dynamics; then a sampled-data predictor-based controller is proposed, and, finally, robustness improvement of the prediction errors is achieved through a suitable redesign.

Section 2 states the problem and specifies the classes of systems under study: nonlinear systems affected by input-delays; strict feedforward-like dynamics, admitting finitely computable predictor map, directly or via preliminary coordinates change and feedback; strict feedback-like systems, characterized by a delay in the connection state state variable. Section 3 reports on the sampled-data predictor based stabilizing controller with discrete-time predictor map. Section 4 discusses a modified sampled-data predictor based controller via Immersion and Invariance (I\&I) design. Section 5 deals with the specific case of a two block cascade dynamics with delay in the state connection variable. Again it is shown how to recast the stabilizing problem in the Immersion and Invariance context. Academic examples illustrate the computational aspects. No complete proofs are reported but adequately referred to in previous authors' work.

\section{Sampled-data models of differential dynamics with delays}

\subsection{The class of systems under study}

We consider nonlinear dynamics over $\mathbb{R}^{n}$ with scalar valued input $u$

$$
\dot{x}(t)=f(x(t), u(t-\tau))
$$

with equilibrium $x_{*}\left(f\left(x_{*}, 0\right)=0\right)$ and known constant delay $\tau \geq 0$; when $\tau=0$ in (1) the so called delay-free dynamics is recovered as

$$
\dot{x}(t)=f(x(t), u(t))
$$


Throughout the paper, maps and vector fields are assumed smooth over the respective definition spaces (i.e. infinitely differentiable $-C^{\infty}$ ) and the delay-free system system corresponding to (1) when $\tau=0$ is forward complete ${ }^{1}$. In (1), $u \in M_{U}^{[-\tau, \infty)}$ where $M_{U}^{I}$ denotes the space of measurable and locally bounded functions $u: I \subset \mathbb{R}^{+} \rightarrow U(u: I \rightarrow U$, with $U \subseteq \mathbb{R}$.

The following standing assumptions are set:

- measures are available only at the sampling instants $t=k \delta ; k \geq 0 ; \delta \in] 0, T$, where $\delta$ is the constant sampling period and $T$ is the maximum allowable sampling period;

- the control is constant over time intervals of length $\delta$; i.e. $u \in \mathscr{U}_{\delta}=\{u \in$ $M_{U}$ s.t. $u(t)=u_{k}, \forall t \in[k \delta,(k+1) \delta[; k \geq 0\}$ (sampled-data control);

- $\delta$ is chosen so that $\tau=N \delta$ for a suitable integer $N$.

- Assumption A - The delay free dynamics associated to (1) is smoothly stabilizable; i. e. there exists a smooth continuous-time feedback $u=\gamma(x)$ with $\gamma\left(x_{*}\right)=0$ and a proper Lyapunov function $V: \mathbb{R}^{n} \rightarrow \mathbb{R}_{\geq 0}$ with $V\left(x_{*}\right)=0$ such that $\dot{V}(x)=\mathrm{L}_{f(\cdot, \gamma(\cdot))} V(x)<0$ and with $\left.\frac{\partial L_{f(\cdot, u)} V(\cdot)}{\partial u}\right|_{u=\gamma(x)} \neq 0$ for all $x \in \mathbb{R}^{n} /\left\{x_{*}\right\}$.

Remark 1. Smoothness of the feedback $\gamma(\cdot)$ is instrumental to computational purposes.

Under these assumptions, the objective of this work is to discuss direct sampleddata strategies which make the the equilibrium $x_{*}$ of (1) S-GAS in the sense of the following definition.

Definition 1. Consider the sampled-data system $\dot{x}=f\left(x, u_{k}\right)$. We say that the closedloop equilibrium of $\dot{x}=f\left(x, \alpha\left(x_{k}\right)\right)$ is sampled-data globally asymptotically stable (S-GAS) for some piecewise constant feedback $u_{k}=\alpha\left(x_{k}\right)$ if the equilibrium of its sampled equivalent $x_{k+1}=F^{\delta}\left(x_{k}, \alpha\left(x_{k}\right)\right)$ is globally asymptotically stable (GAS) at the sampling instants $t=k \delta, k \geq 0$.

Remark 2. The scalar input case is developed for the sake of simplicity but multiinput illustrative examples are reported in [17, 20].

\subsection{Equivalent sampled-data state representations}

As well known, setting $u(t)=u_{k}$ for $t \in[k \delta,(k+1) \delta[$ and assuming $\tau=N \delta$, (1) rewrites as the interconnection of a continuous-time delay free dynamics and a discrete-time finite dimensional linear dynamics; namely, one gets the so-called equivalent hybrid dynamics

\footnotetext{
${ }^{1}$ Assuming the delay free dynamics forward complete ensures that the delayed one (1) is too: $\forall x_{0}$ and $u \in M_{U}^{[-\tau, \infty)}$ the solution $x(t)$ of (1) with initial condition $x(0)=x_{0} \in \mathbb{R}^{n}$ exists $\forall t \geq 0$.
} 


$$
\begin{aligned}
\dot{x}(t) & =f\left(x(t), v_{k}^{1}\right) ; \quad t \in[k \delta,(k+1) \delta[ \\
v_{k+1}^{1}=v_{k}^{2} ; \quad \ldots ; & v_{k+1}^{N}=u_{k}
\end{aligned}
$$

that is now free of delays. By integrating (3a) over $[k \delta,(k+1) \delta[$ and initial condition $x_{k}:=x(k \delta)$, one describes the equivalent sampled-data dynamics in the form of a map (or a difference equation) over $\mathbb{R}^{n} \times \mathbb{R}^{N}$ as

$$
\begin{aligned}
& x_{k+1}=F^{\delta}\left(x_{k}, v_{k}^{1}\right)=\left.e^{\delta \mathrm{L}_{f\left(,, v_{k}^{1}\right)} x}\right|_{x_{k}} \\
& v_{k+1}^{1}=v_{k}^{2} ; \quad \ldots \quad v_{k+1}^{N}=u_{k} .
\end{aligned}
$$

The dynamics (4a) describes the exact sampled-data equivalent of the (3a). In most cases, a closed form of (4a) cannot be computed and the map $F^{\delta}\left(\cdot, v^{1}\right)$ is described by its series expansion ${ }^{2}$ in powers of $\delta$

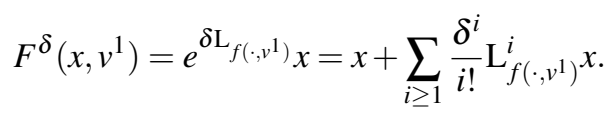

Finite order approximations in $O\left(\delta^{p}\right)^{3}$ are currently used in practice though the above series might have a finite number of terms in $\delta$ (finite discretizability of $\left.f\left(\cdot, v^{1}\right)\right)$ in some specific cases.

Stabilization of the input-delayed dynamics (1) can be thus reformulated in terms of stabilization of the extended dynamics (4) so clearly involving nonlinear discretetime control strategies. However, by exploiting the cascade structure exhibited by (4) and assuming the existence of a discrete-time stabilizing controller for the delay free dynamics (i.e., when $v_{k}^{1}=u_{k}, N=0$ in (4)), predictor-based control strategies with discrete-time prediction map can be worked out.

Remark 3. The case of measurement delays can be treated analogously.

Remark 4. In case of a non entire delays (say $\tau=N \delta+\sigma$ with $\sigma \in] 0, \delta[$ ), the extended sampled dynamics can be defined over $\mathbb{R}^{n} \times \mathbb{R}^{N+1}$ as below 4

$$
\begin{aligned}
& x_{k+1}=F_{1}^{\delta}\left(x_{k}, \sigma, v_{k}^{1}, v_{k}^{2}\right)=e^{\left.\sigma \mathrm{L}_{f\left(,, v_{k}^{1}\right)} \circ e^{(\delta-\sigma) \mathrm{L}_{f\left(,, v_{k}^{2}\right)}} x\right|_{x_{k}}} \\
& v_{k+1}^{1}=v_{k}^{2} ; \quad \ldots \quad ; v_{k+1}^{N}=u_{k} .
\end{aligned}
$$

$F_{1}^{\delta}\left(\sigma, v_{k}^{1}, v_{k}^{2}\right)$ is parameterized by both the fractional part of the delay $\sigma$ and past values of the input variables so that the design strategies developed in the sequel should be modified. The extension of predictor-based techniques to this case has been pro-

${ }^{2} L_{f}$ denotes the Lie derivative operator, $L_{f}=\sum_{i=1}^{n} f_{i}(\cdot) \frac{\partial}{\partial x_{i}} \cdot e^{L_{f}}$ (or $e^{f}$, when no confusion arises) denotes the associated Lie series operator, $e^{\mathrm{L}_{f}}:=1+\sum_{i \geq 1} \frac{L_{f}^{i}}{i !}$.

${ }^{3}$ A function $R(x, \delta)=O\left(\delta^{p}\right)$ is said of order $\delta^{p} ; p \geq 1$ if whenever it is defined it can be written as $R(x, \delta)=\delta^{p-1} \tilde{R}(x, \delta)$ and there exist a function $\theta \in \mathscr{K}_{\infty}$ and $\delta^{*}>0$ s. t. $\forall \delta \leq \delta^{*},|\tilde{R}(x, \delta)| \leq \theta(\delta)$.

$4 \circ$ denotes the composition of operators and functions. 
posed in [21] while an alternative solution relying on the concept of reduction has been discussed in [22].

\subsection{Finite sampling under coordinates change and feedback}

As previously anticipated, the existence of a closed form (possibly finitely computable) expression of the sampled model may be useful. As well known, upper triangular (say strict-feedforward) forms as

$$
\begin{aligned}
\dot{x}_{1}(t)= & f_{1}\left(x_{2}(t), \ldots, x_{n}(t), u(t-\tau)\right) \\
\dot{x}_{2}(t)= & f_{2}\left(x_{3}(t), \ldots, x_{n}(t), u(t-\tau)\right) \\
& \ldots \\
\dot{x}_{n}(t)= & f_{n}(u(t-\tau))
\end{aligned}
$$

where $x_{i} \in \mathbb{R}(i=1, \ldots, n)$ can be finitely integrated through a bottom-up iterative procedure. When assuming the input piecewise constant and affected by delay $\tau=$ $N \delta$, the associated sampled-data model still preserves finite discretizability in $\delta$.

Several mechanical systems exhibit those triangular forms (as the chained forms or their extensions) either from direct modeling or through preliminary coordinates changes and continuous-time feedback [23, 24]. Accordingly, in [25] we examine the conditions allowing finite discretizability through coordinates change and feedback. In the present context, we underline that if such a transformation exists on the delay free dynamics associated to (1), then it can be extended to the input-delayed ones. This fact is easily verified and stated as follows.

Proposition 1. Let the dynamics (1) and consider when $\tau=0$ the delay-free dynamics (2). Assume the existence of coordinates change $\tilde{x}=\phi(x)$ and a state feedback $u(t)=k(x(t), v(t))$ with $v \in M_{U}^{[-\tau, \infty)}$ making (2) finitely discretizable in $\delta$ of order $p$. Then, for $\tau>0$, the retarded feedback $u(t-\tau)=k(x(t), v(t-\tau))$ and the same $\tilde{x}=\phi(x)$ make (1) finitely discretizable at the same order $p$ in $\delta$.

Basically, Proposition 1 implies that the predictor map is finitely computable once a preliminary feedback is applied. Denoting by $\tilde{f}(\tilde{x}, v)$ the continuous-time dynamics in the $(\tilde{x}, v)$ coordinates and by $\tilde{F}^{\delta}(\tilde{x}, v)$ its sampled model, the design is performed in the $\left(\tilde{x_{k}}, v_{k}\right)$ coordinates. Then, the original control is expressed in terms of the computable predictor map $F^{\delta}\left(\cdot, v_{k}\right)$ so getting the piecewise continuous control signal

$$
\begin{aligned}
& \qquad u(k \delta+s)=k\left(x((k+N) \delta+s), v_{k}\right)=\tilde{k}\left(\tilde{x}((k+N) \delta+s), v_{k}\right) \\
& \text { for } s \in\left[0, \delta\left[, k \geq 0 \text { and } \tilde{x}(k \delta+s)=\tilde{F}^{s}\left(\tilde{x}(k \delta), v_{k}\right) \text { for } N=0\right. \text { and }\right. \\
& \tilde{x}((k+N) \delta+s)=\tilde{F}^{s}\left(\cdot, v_{k}\right) \circ \tilde{F}^{\delta}\left(\cdot, v_{k-1}\right) \circ \cdots \circ \tilde{F}^{\delta}\left(\tilde{x}(k \delta), v_{k-N}\right) \quad \text { for } N \geq 1 .
\end{aligned}
$$


Dynamics admitting chained form-like representations generally do not admit continuous-time smooth stabilizing control laws. Thus, sampled-data multirate control strategies have been proposed in [26] to deal with trajectory planning or finitetime tracking objectives. In presence of delays, Proposition 1 suggests to resettle these control problems in the sampled-data context by taking advantage of the simplified transformed dynamics. Arguing so, Assumption A is naturally relaxed and sampling becomes properly an instrumental tool of the design.

\subsubsection{The unicycle, an illustrative example}

Let the kinematics equations of a wheeled vehicle over $\mathbb{R}^{3}$

$$
\dot{x}(t)=v(t) \cos \theta(t) ; \quad \dot{y}(t)=v(t) \sin \theta(t) ; \quad \dot{\theta}(t)=\omega(t)
$$

where $v$ and $\omega$ denote respectively the forward and steering velocities. As well known, the change of coordinates $\left(x_{1}=x \cos \theta+y \sin \theta, x_{2}=\sin \theta-y \cos \theta, x_{3}=\theta\right)$

$$
\left(\begin{array}{lll}
x_{1} & x_{2} & x_{3}
\end{array}\right)^{\top}=\left(\begin{array}{ll}
x \cos \theta+y \sin \theta & \sin \theta-y \cos \theta, \theta
\end{array}\right)^{\top}
$$

and state feedback

$$
u_{1}(t)=v(t)-x_{2}(t) \omega(t) ; \quad u_{2}(t)=\omega(t)
$$

transform the dynamics (6) into the nonholonomic integrator

$$
\dot{x}_{1}(t)=u_{1}(t) ; \quad \dot{x}_{2}(t)=u_{2}(t) x_{1}(t) ; \quad \dot{x}_{3}(t)=u_{2}(t) .
$$

When setting $u_{i}(t)=u_{i k}$ for $t \in\left[k \delta,(k+1) \delta\left[, i=1,2\right.\right.$ and $x_{i k}=x_{i}(k \delta)$ for $i=1,2,3$, one gets an exact sampled equivalent dynamics of finite order 2 in $\delta$; namely,

$$
x_{1 k+1}=x_{1 k}+\delta u_{1 k} ; \quad x_{2 k+1}=x_{2 k}+\delta u_{2 k} x_{1 k}+\frac{\delta^{2}}{2} u_{2 k} u_{1 k} \quad x_{3 k+1}=x_{3 k}+\delta u_{2 k} .
$$

Accordingly, introducing a delay $\tau$ over the inputs, the coordinate change (7) and delayed version of feedback (8) transform (6) into the retarded chained form

$$
\dot{x}_{1}(t)=u_{1}(t-\tau) ; \dot{x}_{2}(t)=u_{2}(t-\tau) x_{1}(t) ; \dot{x}_{3}(t)=u_{2}(t-\tau)
$$

which still admits a sampled model. Assuming now $\tau=\delta$, the sampled-data model in the extended coordinates $\left(x_{1}, x_{2}, x_{3}, w_{1}, w_{2}\right)$ with $w_{i}=u_{i k-1}(i=1,2)$ takes the form

$$
\begin{aligned}
x_{1 k+1}=x_{1 k}+\delta w_{1 k} ; \quad x_{2 k+1} & =x_{2 k}+\delta w_{2 k} x_{1 k}+\frac{\delta^{2}}{2} w_{2 k} w_{1 k} ; \quad x_{3 k+1}=x_{3 k}+\delta w_{2 k} \\
w_{1 k+1} & =u_{1 k} ; \quad w_{2 k+1}=u_{2 k} .
\end{aligned}
$$


Once a control solution over $\left(u_{1}, u_{2}\right)$ is computed, the piecewise continuous controller in terms of $(v, \omega)$ is described for $s \in[0, \delta[$ as

$$
v(k \delta+s)=u_{1 k}+x_{2}(k \delta+\delta+s) u_{2 k} ; \quad \omega(k \delta+s)=u_{2 k}
$$

with $x_{2}(k \delta+\delta+s)=x_{2 k+1}+s u_{2 k} x_{1 k+1}+\frac{\sigma^{2}}{2} u_{2 k} u_{1 k}$, so getting after substitutions

$v(k \delta+s)=u_{1}+x_{2} u_{2}+s x_{1}\left(u_{2}\right)^{2}+\delta x_{1} u_{2} w_{2}+s \delta w_{1}\left(u_{2}\right)^{2}+\frac{s^{2}}{2} u_{1}\left(u_{2}\right)^{2}+\frac{\delta^{2}}{2} u_{2} w_{2} w_{1}$.

We refer the interested reader to [17] for a complete discussion while mechanical structures of this type can be worked out along these lines [20].

\subsection{Cascade dynamics with state delays}

Let us now extend the previous arguments to the case of feedback-like cascade dynamics affected by state delays. Consider the dynamics

$$
\dot{x}_{1}(t)=f_{1}\left(x_{1}(t), x_{2}(t)\right) ; \quad \dot{x}_{2}(t)=u(t)
$$

with $x_{1} \in \mathbb{R}^{n_{1}}, x_{2} \in \mathbb{R}$ and $f_{1}\left(x_{1 *}, x_{2 *}\right)=0$ and assume that a time delay $\tau$ is affecting the connection variable $x_{2}$; i.e.,

$$
\dot{x}_{1}(t)=f_{1}\left(x_{1}(t), x_{2}(t-\tau)\right) ; \quad \dot{x}_{2}(t)=u(t) .
$$

By setting $x_{2}^{r}(t)=x_{2}(t-\tau)$, the delay $\tau$ is moved onto the input variable $u$; namely, one gets

$$
\dot{x}_{1}(t)=f_{1}\left(x_{1}(t), x_{2}^{r}(t)\right) ; \quad \dot{x}_{2}^{r}(t)=u(t-\tau)
$$

that exhibits the form (1) with $x=\left(x_{1}^{\top}, x_{2}^{r}\right)^{\top}$. Analogously, one can consider a delay in the connection variable $x_{2}$ in the feedforward like extension of (9)

$$
\begin{aligned}
\dot{x}_{1}(t)= & f_{1}\left(x_{1}(t), x_{2}(t-\tau)\right) \\
\dot{x}_{2}(t)= & g_{2}\left(x_{2}(t), x_{3}(t-\tau), u(t)\right) \\
& \vdots \\
\dot{x}_{n}(t)= & g_{n}\left(x_{n}(t-\tau), u(t)\right)
\end{aligned}
$$

with $x_{1} \in \mathbb{R}^{n_{1}}, x_{i} \in \mathbb{R}$ for $i=2, \ldots, n$ and $f_{1}\left(x_{1 *}, x_{2 *}\right)=0$. By setting $x_{i}^{r}(t)=x_{i}(t-\tau)$ for $i=2, \ldots, n$, one moves the delay $\tau$ onto the successive interconnecting variable $x_{i+1}$ and onto the input $u$ until one recovers a specific case of (1) with $x=\left(x_{1}, x_{2}^{r}, \ldots, x_{n}^{r}\right)$. In Section 5, we prove sampled-data stabilizability of (10) by assuming the existence of a fictitious feedback law $x_{2}=k\left(x_{1}\right)$ ensuring stabilization of the $x_{1}$-dynamics (thus replacing Assumption $\mathbf{A}$ ). 


\section{Sampled-data predictor-based stabilization}

Consider now the input delayed dynamics (1) with extended sampled equivalent model (4). The design of predictor-based controllers with discrete-time prediction map is described below. We first recall that from Assumption A, one directly infers the existence of a sampled-data feedback $u_{k}=\gamma^{\delta}\left(x_{k}\right)$ stabilizing the delay-free sampled dynamics while guaranteeing, at the sampling instants, the same Lyapunov performances as in continuous time. The following result is recalled from [27].

Theorem 1. (Input-Lyapunov Matching - ILM) Consider the delay free dynamics associated to (1) under A. Then, there exist $T>0$ and for each $\delta$ in $] 0, T$, a sampled feedback $\gamma^{\delta}(x)$ which satisfies the ILM equality

$$
V\left(F^{\delta}\left(x_{k}, u_{k}\right)\right)-V\left(x_{k}\right)=\int_{k \delta}^{(k+1) \delta} \mathrm{L}_{f(\cdot, \gamma(\cdot))} V(x(s)) d s
$$

i.e. S-GAS is yielded by $u_{k}=\gamma^{\delta}\left(x_{k}\right)$.

The proof is constructive and $\gamma^{\delta}(\cdot)$ is described by its series expansion around $\gamma(x)$; i.e. $\gamma^{\delta}(x)=\gamma(x)+\sum_{i \geq 1} \frac{\delta^{i}}{(i+1) !} \gamma_{i}(x)$ with $\gamma^{\delta}\left(x_{*}\right)=0$. This result is developed in [28] when considering $f(x, u)=f(x)+u g(x)$ with constructive algorithms for computing the feedback solution.

Stabilization of the input delayed dynamics (4) follows through state prediction; i.e. $u_{k}=\gamma^{\delta}\left(x_{k+N}\right)$ with $N$-steps ahead prediction and suitably chosen initial conditions.

Theorem 2. Consider (1) under the assumptions of Theorem 1. Then, $x_{k}^{p}:=F^{\delta}\left(\cdot, u_{k-N}\right) \circ$ $\ldots \circ F^{\delta}\left(x_{k}, u_{k-1}\right)$ is a predictor for (1) with dynamics

$$
x_{k+1}^{p}=F^{\delta}\left(x_{k}^{p}, u_{k}\right)
$$

and initial conditions $x_{0}^{p}=F^{\delta}\left(\cdot, u_{-N}\right) \circ \ldots \circ F^{\delta}\left(x_{0}, u_{-1}\right)$. Moreover, the feedback $u_{k}=\gamma^{\delta}\left(x_{k}^{p}\right)$ makes the closed-loop equilibrium of (1) $S$-GAS.

The above result underlines how sampled-data stabilizability is directly ensured by smooth stabilizability of the continuous-time delay-free dynamics. Contrarily to existing works dealing with sample-and-hold solutions, the proposed compensating feedback is based on a two step redesign procedure: first, for $\tau=0$, a sampleddata feedback ensuring GAS in closed-loop is constructed through Input Lyapunov Matching (ILM); then, the final sampled-data stabilizing predictor-based feedback for the retarded dynamics is built by defining a discrete-time predictor dynamics.

Remark 5. Contrarily to the predictor-based techniques proposed in [10, 12], we describe a discrete-time prediction map (14) that exploits the piecewise constant nature of the input. This implies that prediction of the full state at any time instant $t$ is unnecessary for stabilizing purposes so overcoming some tough numerical issues. 


\section{Immersion and Invariance stabilization with input delays}

As well known, predictor-based techniques strongly suffer from robustness to prediction errors. In this context, stabilization through invariant sets offers interesting refinements to Lyapunov-based control by exploiting the system structure. Taking advantage of the underlying cascade of (4), in [19] we have shown that Immersion and Invariance [29, 30] provides a natural set-up for input-delayed dynamics under sampling. More in detail, the delay-free stable closed loop dynamics identifies the target systems evolving over a stable manifold. Accordingly, I\&I stabilization requires to drive the off-the manifold trajectories to zero with boundedness of the full state ones. Manifold invariance guarantees that the on-the-manifold closed-loop dynamics recover the predictor evolutions defining the target. The I\&I feedback modifies the predictor-based one so preventing from big control effort and improving robustness when predictor-based controllers cannot be exactly computed.

\subsection{I\&I stabilization}

Immersion and Invariance was firstly introduced in continuous time in [29] and then proposed for discrete-time adaptive control in [31]. It is reformulated below in the discrete-time and sampled contexts for completeness (see also [30, 32, 33]).

Theorem 3. Consider a nonlinear discrete-time dynamics in the form of a map

$$
x_{k+1}=F\left(x_{k}, u_{k}\right)
$$

with $x \in \mathbb{R}^{n}, u \in \mathbb{R}$ and equilibrium state $x_{*}$ to be stabilized. Assume that there exists a $p<n$ so that there exist mappings $\alpha(\cdot): \mathbb{R}^{p} \rightarrow \mathbb{R}^{p}, \pi(\cdot): \mathbb{R}^{p} \rightarrow \mathbb{R}^{n}, c(\cdot): \mathbb{R}^{p} \rightarrow \mathbb{R}$, $\phi(\cdot): \mathbb{R}^{n} \rightarrow \mathbb{R}^{n-p}$ and $\psi(\cdot, \cdot): \mathbb{R}^{n \times(n-p)} \rightarrow \mathbb{R}$ such that the following four conditions hold:

$\left.\boldsymbol{I}_{1}\right)$ the target dynamics $\xi_{k+1}=\alpha\left(\xi_{k}\right)$ with $\xi \in \mathbb{R}^{p}$ has a GAS equilibrium at $\xi_{*}$ and $x_{*}=\pi\left(\xi_{*}\right)$;

I $) \quad$ There exists $c(\cdot): \mathbb{R}^{p} \rightarrow \mathbb{R}$ such that $F\left(\pi\left(\xi_{k}\right), c\left(\xi_{k}\right)\right)=\pi\left(\alpha\left(\xi_{k}\right)\right)$;

$\left.\boldsymbol{I}_{3}\right)$ The following set-identity holds:

$$
\left\{x \in \mathbb{R}^{n} \mid \phi(x)=0\right\}=\left\{x \in \mathbb{R}^{n} \mid x=\pi(\xi) \quad \text { for } \quad \xi \in \mathbb{R}^{p}\right\} .
$$

$\left.\boldsymbol{I}_{4}\right) \quad$ Setting $z=\phi(x)$ with $z_{0}=\phi\left(x_{0}\right)$, the trajectories of

$$
z_{k+1}=\phi\left(F\left(x_{k}, \psi\left(x_{k}, z_{k}\right)\right)\right) ; \quad x_{k+1}=F\left(x_{k}, \psi\left(x_{k}, z_{k}\right)\right)
$$

are bounded and satisfy $\lim _{k \rightarrow \infty} z_{k}=0$ and $\psi(\pi(\xi), 0)=c(\xi)$.

Then, $x_{*}$ is a globally asymptotically stable equilibrium of the closed loop dynamics

$$
x_{k+1}=F\left(x_{k}, \psi\left(x_{k}, \phi\left(x_{k}\right)\right)\right) .
$$


Definition 2. Any discrete-time system (15) satisfying conditions $\mathrm{I}_{1}$ to $\mathrm{I}_{4}$ of Theorem 3 is said $I \& I$ stabilizable with target dynamics $\xi_{k+1}=\alpha\left(\xi_{k}\right)$. Accordingly, the delay free continuous-time dynamics of (1) is said sampled-data I\&I stabilizable if its sampled equivalent dynamics $x_{k+1}=F^{\delta}\left(x_{k}, u_{k}\right)=\left.e^{\delta f\left(\cdot, u_{k}\right)} x\right|_{x_{k}}$ with $u \in \mathscr{U}_{\delta}$ is I\&I stabilizable in the discrete-time sense ${ }^{5}$.

Definition 3. The continuous-time dynamics $\dot{x}=f(x, u)$ is said sampled-data $I \& I$ stabilizable if its sampled equivalent dynamics $x_{k+1}=F^{\delta}\left(x_{k}, u_{k}\right)=\left.e^{\delta f\left(\cdot, u_{k}\right)} x\right|_{x_{k}}$ with $u \in \mathscr{U}_{\delta}$ is I\&I stabilizable in the sense of Definition 2.

\subsection{I\&I stabilization with input delays}

Further exploiting the structure of the extended sampled model (4), we show that Assumption A implies its I\&I stabilizability. We are now extending the result in [19] to more general systems of the form (1). Let us rewrite (4) in compact form as

$$
x_{k+1}^{e}=F_{e}^{\delta}\left(x_{k}^{e}, u_{k}\right)
$$

with $x^{e}=\left(x^{\top}, \bar{v}^{\top}\right) \in \mathbb{R}^{n+N}, \bar{v}=\left(v^{1}, \ldots, v^{N}\right)^{\top} \in \mathbb{R}^{N}$ and equilibrium $x_{*}^{e}=\left(x_{*}^{\top}, 0\right)^{\top}$. According to Theorem 3, one defines the target dynamics

$$
x_{k+1}=\alpha^{\delta}\left(x_{k}\right):=F^{\delta}\left(x_{k}, \gamma^{\delta}\left(x_{k}\right)\right)
$$

whose equilibrium $x_{*}$ is GAS by construction of $\gamma^{\delta}(\cdot)$ in Theorem 1 . Setting now

$$
\bar{z}:=\left(z^{1}, \cdots, z^{N}\right)^{\top}:=\phi^{\delta}\left(x^{e}\right)=\left(\phi_{1}^{\delta}\left(x^{e}\right), \ldots, \phi_{N}^{\delta}\left(x^{e}\right)\right)^{\top}
$$

with

$$
\phi_{i}^{\delta}\left(x_{k}, \bar{v}_{k}\right):=v_{k}^{i}-\gamma^{\delta}\left(x_{k+i-1}\right), \quad x_{k+i}=\left.\underbrace{e^{\delta f\left(\cdot, v_{k}^{1}\right)} \circ \ldots \circ e^{\delta f\left(\cdot, v_{k}^{i-1}\right)}}_{i \text { times }} x\right|_{x_{k}} .
$$

and $\pi^{\delta}(\cdot):=\left(\cdot, \gamma^{\delta}(\cdot), \ldots, \gamma^{\delta}\left(\left(\alpha^{\delta}\right)^{N-1}(\cdot)\right)\right)^{\top}$, the following result can be stated.

Theorem 4. Let the continuous-time input delayed dynamics (1) satisfy Assumption $\mathbf{A}$ and the delay-free stabilizer $\gamma^{\delta}(\cdot)$ be the solution of (13). Then (1) is sampleddata I\&I stabilizable; i.e. its sampled equivalent dynamics (17) is I\&I stabilizable with target dynamics (18).

Consider $\gamma^{\delta}(\cdot)$ as defined in (13) with control Lyapunov function $V(\cdot)$. Computing now $\pi^{\delta}(\cdot): \mathbb{R}^{n} \rightarrow \mathbb{R}^{n+N}$ as above, it is a matter of computations to verify that conditions $I_{1}$ to $I_{3}$ of Theorem 3 are satisfied by construction with $\phi^{\delta}(\cdot)$ described in (19) and

\footnotetext{
${ }^{5}$ Mappings and dynamics are parameterized by $\delta$ as indicated with superscript $(\cdot)^{\delta}$.
} 


$$
c^{\delta}\left(x_{k}\right)=\gamma^{\delta}\left(x_{k+N}\right)=\gamma^{\delta} \circ \underbrace{\alpha^{\delta} \circ \cdots \circ \alpha^{\delta}}_{N \text { times }}\left(x_{k}\right) .
$$

On these bases and from Theorem 3, one directly infers that any feedback $u=$ $\psi^{\delta}(x, \bar{v}, \bar{z})$ such that $\psi^{\delta}\left(\pi^{\delta}(x), 0\right)=c^{\delta}(x)$ and ensuring I\&I stabilization also achieves GAS of the equilibrium of

$$
x_{k+1}=F^{\delta}\left(x_{k}, v_{k}^{1}\right) ; \quad v_{k+1}^{1}=v_{k}^{2} ; \quad \ldots \quad ; v_{k+1}^{N-1}=v_{k}^{N} ; \quad v_{k+1}^{N}=\psi^{\delta}\left(x_{k}, \bar{v}_{k}, \bar{z}_{k}\right)
$$

whenever it is designed to drive $\bar{z}$ to zero with boundedness of the state trajectories of the extended dynamics

$$
\begin{aligned}
z_{k+1}^{1} & =z_{k}^{2} ; \quad \ldots \quad ; z_{k+1}^{N-1}=z_{k}^{N} ; \quad z_{k+1}^{N}=u_{k}-\gamma^{\delta}\left(x_{k+N}\right) \\
x_{k+1} & =F^{\delta}\left(x_{k}, v_{k}^{1}\right) \\
v_{k+1}^{1} & =v_{k}^{2} ; \quad \ldots \quad ; v_{k+1}^{N-1}=v_{k}^{N} ; \quad v_{k+1}^{N}=u_{k} .
\end{aligned}
$$

As a consequence of the structure of (21), condition $\mathrm{I}_{4}$ of Theorem 3 relaxes to requiring $\lim _{k \rightarrow \infty} \psi^{\delta}\left(x_{k}, \bar{v}_{k}, \bar{z}_{k}\right)-\psi^{\delta}\left(x_{k}, \bar{v}_{k}, 0\right)=0$ with $\psi^{\delta}\left(x_{k}, \bar{v}_{k}, 0\right)=\gamma^{\delta} \circ\left(\alpha^{\delta}\right)^{N}\left(x_{k}\right)$. In [19], a multi-rate control strategy is proposed to for $\bar{z} \equiv 0$ to 0 in exactly $N$ steps. However, for implementation issues, an approximate controller of the form

$$
u_{k}=\gamma^{\delta} \circ\left(\alpha^{\delta}\right)^{N}\left(x_{k}\right)+L^{\delta}\left(x_{k}\right) \bar{z}_{k}
$$

with suitably defined gain matrix $\left|L^{\delta}(x)\right|<1$ is needed. The approximate feedback (22) achieves, at least locally, asymptotic stabilization of the equilibrium with improved robustness performances with respect to uncertainties on the delay length or discarded higher order components in the predictor dynamics. As a matter of fact, I\&I introduces a feedback over the prediction error while prediction-based feedback usually work in open-loop (see [19] for a more complete discussion).

Remark 6. When the stable manifold is reached (i.e., $\bar{z}=0), c^{\delta}(x)$ in (20) recovers the $N$-steps ahead predictor-based feedback $\gamma^{\delta} \circ\left(\alpha^{\delta}\right)^{N}(x)$ while in the delay free case $(N=0), c^{\delta}(x)$ reduces to the original ILM-based feedback $\gamma^{\delta}(x)$.

Remark 7. When $N=1$, the I\&I feedback recalls the first step of a backstepping design with Lyapunov function $\bar{V}(x, z)=V(x)+\frac{1}{2} z^{2}$. When $N>1$, the $N$-steps ahead I\&I strategy recalls the $N$-rate backstepping design developed in [28].

\subsubsection{An example}

The following two block cascade system is exploited as an illustrative example throughout the paper. Let

$$
\dot{x}_{1}(t)=x_{1}(t) x_{2}(t) ; \quad \dot{x}_{2}(t)=u(t)
$$


be stabilized by the feedback $u=-2 x_{2}-x_{1}^{2} e^{2 x_{2}}$ and Lyapunov function $V(x)=$ $\frac{1}{2}\left(x_{1}^{2} e^{2 x_{2}}+x_{2}^{2}\right)$. The exact sampled model of (23) takes the form

$$
x_{1 k+1}=e^{\delta\left(x_{2 k}+\frac{\delta}{2} u_{k}\right)} x_{1 k} ; \quad x_{2 k+1}=x_{2 k}+\delta u_{k} .
$$

and a stabilizing sampled-data controller can be computed through ILM design as

$$
u_{k}=\gamma^{\delta}\left(x_{k}\right)=-2 x_{2 k}\left(1-\frac{\delta}{2}\right)-x_{1 k}^{2} e^{2 x_{2 k}}\left(1-\frac{\delta}{2}\left(1+x_{1 k}^{2} e^{2 x_{2 k}}\right)+O\left(\delta^{2}\right)\right.
$$

which is approximated in $O\left(\delta^{2}\right)$. Assuming now an input delay equal to $\delta$ (i.e. $u(t-\tau)=u(t-\delta))$ in (23), one defines the extended sampled equivalent dynamics

$$
x_{1 k+1}=e^{\delta\left(x_{2 k}+\frac{\delta}{2} v_{k}\right)} x_{1 k} ; \quad x_{2 k+1}=x_{2 k}+\delta v_{k} ; \quad v_{k+1}=u_{k} .
$$

Accordingly, the sampled predictor-based feedback is $u_{k}=\gamma^{\delta}\left(x_{k}^{p}\right)$ with $x_{k}^{p}=x_{k+1}$ :

$$
\gamma^{\delta}\left(x^{p}\right)=-2 a^{\delta}(x)-b^{\delta}(x)+\frac{\delta}{2}\left(2 a^{\delta}(x)+b^{\delta}(x)\left(1+b^{\delta}(x)\right)\right.
$$

with $a^{\delta}(x)=x_{2}+\delta \gamma^{\delta}(x) ; b^{\delta}(x)=e^{2\left(x_{2}+\gamma^{\delta}(x)\right)} e^{2 \delta\left(x_{2}+\frac{\delta}{2} \gamma^{\delta}(x)\right)} x_{1}^{2}$. As discussed, a modified I\&I feedback with sampled-data target dynamics

$$
x_{1 k+1}=e^{\delta\left(x_{2 k}+\frac{\delta}{2} \gamma^{\delta}\left(x_{k}\right)\right)} x_{1 k} ; \quad x_{2 k+1}=x_{2 k}+\delta \gamma^{\delta}\left(x_{k}\right) .
$$

and off-the-manifold component $z=v-\gamma^{\delta}(x)$, can be designed to bring $z$ to zero

$x_{1 k+1}=e^{\delta\left(x_{2 k}+\frac{\delta}{2} \gamma^{\delta}\left(x_{k}\right)+\frac{\delta}{2} z_{k}\right)} x_{1 k} ; \quad x_{2 k+1}=x_{2 k}+\delta \gamma^{\delta}\left(x_{k}\right)+\delta z_{k} ; \quad z_{k+1}=u_{k}-\gamma^{\delta}\left(x_{k+1}\right)$.

Accordingly, one computes (omitting the ${ }_{k}$-dependency)

$\psi^{\delta}(x)=-2 a^{\delta}(x)-2 \delta z-b^{\delta}(x) e^{3 \delta z}+\frac{\delta}{2}\left(2 a^{\delta}(x)+2 \delta z+b^{\delta}(x) e^{3 \delta z}\left(1+b^{\delta}(x) e^{3 \delta z}\right)\right.$.

so recovering $\gamma^{\delta}\left(x_{k}^{p}\right)$ when $z=0$ with robustness improvement when $z \neq 0$.

\section{Sampled-data I\&I stabilization with state delays}

In this section, stabilization of the strict-feedback dynamics (10) with delays on the connecting state variable is addressed. Since (10) exhibits the form (1), the former arguments still apply for sampled stabilization when setting as extended state $x=\left(x_{1}^{T}, x_{2}\right)^{T}$. A different solution exploiting the interconnection structure of (9) is described below when reformulating Assumption A over $f_{1}(\cdot)$ in (9) as follows. 
Assumption B - There exists a smooth mapping $x_{2}(t)=k\left(x_{1}\right)$ with $k\left(x_{1 *}\right)=x_{2 *}$ and a proper Lyapunov function $W: \mathbb{R}^{n_{1}} \rightarrow \mathbb{R}_{\geq 0}$ such that $\dot{W}\left(x_{1}\right)=\mathrm{L}_{f\left(x_{1}, k\left(x_{1}\right)\right)} W\left(x_{1}\right)<0$ and $\left.\frac{\partial L_{f_{1}} W}{\partial x_{1}}\right|_{x_{2}=k\left(x_{1}\right)} \neq 0$ for any $x_{1} \neq x_{1 *}$.

Remark 8. Through easy backstepping-like arguments, one can prove that Assumption B implies Assumption A.

Consider (9) under Assumption B (equivalently (11) when setting $x_{2}^{r}(t)=x_{2}(t-\tau)$ ) with sampled equivalent dynamics described over $\mathbb{R}^{n_{1}+1} \times \mathbb{R}^{N}$ as

$$
x_{1 k+1}=F_{1}^{\delta}\left(x_{1 k}, x_{2 k}^{r}, v_{k}^{1}\right) ; \quad x_{2 k+1}^{r}=x_{2 k}^{r}+\delta v_{k}^{1} ; \quad v_{k+1}^{1}=v_{k}^{2} ; \quad \ldots ; v_{k+1}^{N}=u_{k}
$$

or, in a more compact form, as $x_{k+1}^{e}=F_{e}^{\delta}\left(x_{k}^{e}, u_{k}\right)$ with $x^{e}=\left(x_{1}^{\top}, x_{2}^{r}, v^{1}, \ldots, v^{N}\right)^{\top}$.

Two preliminary results are instrumental for extending to (9) the results provided in $[32,33]$ for delay-free input-affine systems. More in detail, we show what follows.

- When $\tau=0$, Assumption B implies I\&I stabilizability of the delay free dynamics (9) which implies I\&I stabilizability of its equivalent sampled model (Proposition 2);

- Sampled I\&I stabilizability of the delay-free (9) implies sampled-data I\&I stabilizability of the retarded (10) (Theorem 5).

Proposition 2. Let (9) satisfy Assumption B. Then, its sampled equivalent dynamics

$$
x_{1 k+1}=F_{1}^{\delta}\left(x_{1 k}, x_{2 k}, u_{k}\right) ; \quad x_{2 k+1}=x_{2 k}+\delta u_{k}
$$

is I\&I stabilizable with target dynamics

$$
x_{1 k+1}=F_{1}^{\delta}\left(x_{1 k}, k^{\delta}\left(x_{1 k}\right), \gamma^{\delta}\left(x_{1 k}\right)\right)
$$

where $k^{\delta}=k+\sum_{i \geq 1} \frac{\delta^{i}}{(i+1) !} k_{i}$ and $\gamma^{\delta}=\dot{k}+\sum_{i \geq 1} \frac{\delta^{i}}{(i+1) !} \gamma_{i}$ are solutions of the equalities

$$
\begin{aligned}
& W\left(x_{1 k+1}\right)=W\left(x_{1 k}\right)+\int_{k \delta}^{(k+1) \delta} L_{f(\cdot, k(\cdot))} W\left(x_{1}(\tau)\right) d \tau \\
& k^{\delta}\left(x_{1 k+1}\right)=k^{\delta}\left(x_{1 k}\right)+\delta \gamma^{\delta}\left(x_{1 k}\right) .
\end{aligned}
$$

Equality (30a) ensures ILM at the sampling instants of the closed loop Lyapunov function $W\left(x_{1}\right)$ on the target dynamics (29) and, hence, the stability of its closedloop equilibrium. Equality (30b) guarantees invariance of the corresponding manifold that is implicitly defined by the condition $z=x_{2}-k^{\delta}\left(x_{1}\right)=\phi^{\delta}\left(x_{1}, x_{2}\right) \equiv 0$. On these bases, the sampled I\&I stabilizing delay-free feedback $u=\psi^{\delta}(x, z)$ is designed to drive $z$ to zero while preserving boundedness of the complete state trajectories

$$
x_{1 k+1}=F_{1}^{\delta}\left(x_{1 k}, x_{2 k}, u_{k}\right) ; x_{2 k+1}=x_{2 k}+\delta u_{k} ; z_{k+1}=z_{k}+\delta u_{k}-k^{\delta}\left(x_{1 k+1}\right)+k^{\delta}\left(x_{1 k}\right) .
$$


GAS of the closed-loop $x$-dynamics follows (delay free case) with

$$
\psi^{\delta}\left(x_{1}, k^{\delta}\left(x_{1}\right), 0\right)=\gamma^{\delta}\left(x_{1}\right)=\frac{1}{\delta}\left(k^{\delta}\left(F_{1}^{\delta}\left(x_{1 k}, k^{\delta}\left(x_{1 k}\right), \gamma^{\delta}\left(x_{1 k}\right)\right)\right)-k^{\delta}\left(x_{1 k}\right)\right)
$$

when $z=0$ as implied by (30b).

Remark 9. The existence of solutions to equalities (30) is guaranteed by the cascade structure of (9) and $\left.\frac{\partial L_{f_{1}} W}{\partial x_{1}}\right|_{x_{2}=k\left(x_{1}\right)} \neq 0, \forall x_{1} \neq x_{1 *}$.

Remark 10. It is a matter of computations to verify that the pair $(k, \dot{k})$ satisfies equalities (30) in $O\left(\delta^{2}\right)$ so emphasizing the fact that the sampled-data pair $\left(k^{\delta}, \gamma^{\delta}\right)$ is computed around the continuous-time solution $(k, \dot{k})$.

The following result generalizes Theorem 3.1 in [18]

Theorem 5. Consider the continuous-time dynamics (10) with $\tau=N \delta$. Suppose that when $\tau=0$, (10) satisfies Assumption $\mathbf{B}$ with $\left.\frac{\partial L_{f_{1}} W}{\partial x_{1}}\right|_{x_{2}=k\left(x_{1}\right)} \neq 0, \forall x_{1} \neq x_{1 *}$. Then, the extended sampled equivalent dynamics (28) is I\&I stabilizable with target dynamics (29).

The above result follows when defining the pair $\left(k^{\delta}, \gamma^{\delta}\right)$ as in Proposition 2. Accordingly, the subsequent extended immersion mapping $\bar{\pi}^{\delta}: \mathbb{R}^{n_{1}} \rightarrow \mathbb{R}^{n_{1}+1} \times \mathbb{R}^{N}$ takes the form

$$
\bar{\pi}^{\delta}\left(x_{1 k}\right)=\left(x_{1 k}^{\top}, k^{\delta}\left(x_{1 k}\right), \gamma^{\delta}\left(x_{1 k}\right), \ldots, \gamma^{\delta}\left(x_{1 k+N-1}\right)\right)^{\top}
$$

while the extended $\bar{\phi}^{\delta}: \mathbb{R}^{n_{1}+1} \times \mathbb{R}^{N} \rightarrow \mathbb{R}^{N+1}$ with $\bar{v}=\left(v^{1}, \ldots, v^{N}\right)^{\top}$ is provided by

$$
\begin{gathered}
z_{1 k}=\bar{\phi}_{1}^{\delta}\left(x_{1 k}, x_{2 k}^{r}, v_{k}\right)=x_{2 k}^{r}-k^{\delta}\left(x_{1 k}\right) ; \quad z_{2 k}=\bar{\phi}_{2}^{\delta}\left(x_{1 k}, x_{2 k}^{r}, v_{k}\right)=v_{k}^{1}-\gamma^{\delta}\left(x_{1 k}\right) \\
\ldots \\
z_{N+1 k}=\bar{\phi}_{N+1}^{\delta}\left(x_{1 k}, x_{2 k}^{r}, v_{k}\right)=v_{k}^{N}-\gamma^{\delta}\left(x_{1 k+N-1}\right) .
\end{gathered}
$$

On these bases, I\&I stabilization of (10) at the sampling instants is achieved by any feedback $u_{k}=\bar{\psi}^{\delta}\left(x_{1 k}, x_{2 k}^{r}, \bar{z}_{k}\right)$ satisfying $\bar{\psi}^{\delta}\left(x_{1}, k^{\delta}\left(x_{1}\right), 0\right)=\gamma^{\delta}\left(x_{1}\right)$ and designed to bring $\bar{z}$ to zero with boundedness of the trajectories of the $\left(x_{1}^{\top}, x_{2}, \bar{z}\right)$-dynamics. We showed in [33] that multi-rate digital control strategies of order the dimension of $\bar{z}$, the off-the manifold component are suitable to bring $\bar{z}$ with invariance.

\subsection{Example}

Consider again the system (23) but now notice that for $x_{2}=-\frac{1}{2} x_{1}^{2}$ the sub-system $\dot{x}_{1}=-\frac{1}{2} x_{1}^{3}$ has a GAS equilibrium at the origin with Lyapunov function $V_{0}\left(x_{1}\right)=$ $\frac{1}{2} x_{1}^{2}$. Suppose now that a delay $\tau$ is acting on the transmission variable $x_{2}$, namely, 


$$
\dot{x}_{1}(t)=x_{1}(t) x_{2}(t-\tau), \quad \dot{x}_{2}(t)=u(t) .
$$

Under state transformation $x_{2}^{r}(t)=x_{2}(t-\tau)$ and dynamical extension, one gets

$$
\dot{x}_{1}(t)=x_{1}(t) x_{2}^{r}(t) ; \quad \dot{x}_{2}^{r}(t)=v_{k} ; \quad v_{k+1}=u_{k}
$$

with exact (single-rate) sampled equivalent model

$$
x_{1 k+1}=e^{\delta\left(x_{2 k}^{r}+\frac{\delta}{2} v_{k}\right)} x_{1 k} ; \quad x_{2 k+1}^{r}=x_{2 k}^{r}+\delta v_{k} ; \quad v_{k+1}=u_{k}
$$

Accordingly, I\&I applies to the above system by setting the target dynamics as $x_{1 k+1}=e^{\delta\left(k^{\delta}\left(x_{1 k}\right)+\frac{\delta}{2} c^{\delta}\left(x_{1 k}\right)\right.} x_{1 k}$ with the solutions to (30a-30b) in $O\left(\delta^{4}\right)$ as

$$
k^{\delta}\left(x_{1}\right)=-\frac{1}{2} x_{1}^{2}-\frac{\delta^{2}}{6}\left(x_{1}^{2}+\frac{1}{8}\right) x_{1}^{4}+O\left(\delta^{3}\right) \quad c^{\delta}\left(x_{1}\right)=\frac{1}{2} x_{1}^{4}+\delta\left(\frac{1}{8}-x_{1}^{2}\right) x_{1}^{4}+O\left(\delta^{2}\right) .
$$

Setting $z_{1}:=x_{2}-k^{\delta}\left(x_{1}\right), z_{2}:=v-c^{\delta}\left(x_{1}\right)$ and $u_{i k}=u\left(k \delta+\frac{(i-1)}{2} \delta\right)$ for $i=1,2$, one computes the double-rate equivalent model as

$$
\begin{aligned}
& x_{1 k+1}=e^{\frac{\delta}{2}\left(2\left(z_{1}+k^{\frac{\delta}{2}}\left(x_{1 k}\right)\right)+\frac{3 \delta}{4}\left(z_{2}+c^{\frac{\delta}{2}}\left(x_{1 k}\right)\right)+\frac{\delta}{4} u_{k}^{1}\right)} x_{1 k} ; \quad x_{2 k+1}^{r}=x_{2 k}^{r}+\frac{\delta}{2}\left(v_{k}+u_{k}^{1}\right) ; \quad v_{k+1}=u_{k}^{2} \\
& z_{1 k+1}=z_{1 k}+k^{\frac{\delta}{2}}\left(x_{1 k}\right)-k^{\frac{\delta}{2}}\left(x_{1 k+1}\right)+\frac{\delta}{2}\left(z_{2}+c^{\frac{\delta}{2}}\left(x_{1 k}\right)+u_{1 k}\right), \quad z_{2 k+1}=u_{2 k}-c^{\frac{\delta}{2}}\left(x_{1 k+1}\right) .
\end{aligned}
$$

Accordingly, the feedback $u=\operatorname{col}\left(u_{1}, u_{2}\right)$ solution to

$$
\frac{\delta}{2} u_{1}=k^{\frac{\delta}{2}}\left(x_{1 k+1}\right)-k^{\frac{\delta}{2}}\left(x_{1 k}\right)-\frac{\delta}{2}\left(z_{2}+c^{\frac{\delta}{2}}\left(x_{1}\right)\right), \quad u_{2}=c^{\frac{\delta}{2}}\left(x_{1 k+1}\right)
$$

guarantees I\&I stabilization in closed-loop.

\section{Conclusion}

The paper revisits recent authors' works by emphasizing the role of sampling for stabilizing nonlinear time-delay systems. While assuming entire delays, we show that smooth stabilizability of the continuous-time delay-free system is enough for deducing the existence of a stabilizer for the retarded dynamics. The proposed solution employs a discrete-time predictor which is instrumental for designing the stabilizing feedback. Finally, a robust redesign is carried out by extending the Immersion and Invariance approach to time-delay systems. The proposed designs are constructive. Perspectives are opened toward more general classes of time-delay systems (non-entire, distributed and multi-channel delays) together with a suitable comparison with reduction-based techniques. 


\section{References}

1. M. Krstic, Delay compensation for nonlinear, adaptive, and PDE systems. Springer, 2009.

2. W. Michiels and S. Niculescu, Stability, control, and computation for time-delay systems. An eigenvalue based approach. Advances in Design and Control, Philadelphia: SIAM Society for Industrial and Applied Mathematics, 2014.

3. M. Krstic, "Input delay compensation for forward complete and strict-feedforward nonlinear systems," IEEE Transactions on Automatic Control, vol. 55, no. 2, pp. 287-303, 2010.

4. F. Mazenc, S. Niculescu, and M. Bekaik, "Backstepping for nonlinear systems with delay in the input revisited," SIAM Journal on Control and Optimization, vol. 49, no. 6, pp. 2239-2262, 2011.

5. N. Bekiaris-Liberis and M. Krstic, "Compensation of state-dependent input delay for nonlinear system," IEEE Transactions on Automatic Control, vol. 58, no. 2, pp. 275-289, 2013.

6. E. Fridman, Introduction to Time-Delay Systems: Analysis and Control. Systems \& Control: Foundations \& Applications, E. Fridman, 2014.

7. P. Pepe, "Robustification of nonlinear stabilizers in the sample-and-hold sense," Journal of the Franklin Institute, vol. 352, no. 10, pp. $4107-4128,2015$.

8. E. Fridman., "A refined input delay approach to sampled-data control," Automatica, vol. 46, no. 2, pp. 421-427, 2010.

9. F. Mazenc and D. Normand-Cyrot, "Reduction model approach for linear systems with sampled delayed inputs," IEEE Transactions on Automatic Control, vol. 58, no. 5, pp. 1263 1268, 2013.

10. I. Karafyllis and M. Krstic, "Nonlinear stabilization under sampled and delayed measurements, and with inputs subject to delay and zero-order hold," IEEE Transactions on Automatic Control, vol. 57, no. 5, pp. 1141-1154, 2012.

11. F. Mazenc, M. Malisoff, and T. N. Dinh, "Robustness of nonlinear systems with respect to delay and sampling of the controls," Automatica, vol. 49, no. 6, pp. 1925-1931, 2013.

12. I. Karafyllis and M. Krstic, "Numerical schemes for nonlinear predictor feedback," Mathematics of Control, Signals, and Systems, vol. 26, pp. 519-546, 2014.

13. P. Pepe, "Stabilization in the sample-and-hold sense of nonlinear retarded systems," SIAM Journal on Control and Optimization, vol. 52, no. 5, pp. 3053-3077, 2014.

14. F. Mazenc and E. Fridman, "Predictor-based sampled-data exponential stabilization through continuous-discrete observers," Automatica, vol. 63, pp. 74-81, 2016.

15. P. Pepe and E. Fridman, "On global exponential stability preservation under sampling for globally lipschitz time-delay systems," Automatica, vol. 82, no. 8, pp. 295 - 300, 2017.

16. S. Monaco, D. Normand-Cyrot, and V. Tanasa, "Digital stabilization of input delayed strict feedforward dynamics," in Proc. 51st IEEE-CDC, Maui, Hawaii, pp. 7535-7540, 2012.

17. V. Tanasa, S. Monaco, and D. Normand-Cyrot, "Digital stabilization of finite sampled nonlinear dynamics with delays: the unicycle example,", in Proc. ECC'13, pp. 2591-2596, 2013.

18. M. Mattioni, S. Monaco, and D. Normand Cyrot, "Sampled-data stabilisation of a class of state-delayed nonlinear dynamics," in 54th IEEE Conference on Decision and Control (CDC), pp. 5695-5700, 2015.

19. S. Monaco, D. Normand-Cyrot, and M. Mattioni, "Sampled-data stabilization of nonlinear dynamics with input delays through immersion and invariance," IEEE Transactions on Automatic Control, vol. 62, no. 5, pp. 2561-2567, 2016.

20. L. Celsi, R. Bonghi, S. Monaco, and D. Normand-Cyrot, "On the exact steering of finite sampled nonlinear dynamics with input delays," IFAC-PapersOnLine, vol. 48, no. 11, pp. 674 679, 2015.

21. M. Mattioni, S. Monaco, and D. Normand-Cyrot, "Further results on sampled-data stabilization of time-delay systems," in Proceedings of the 20th IFAC World Congress, pp. 1491514920, 2017.

22. M. Mattioni, S. Monaco, and D. Normand-Cyrot, "Sampled-data reduction of nonlinear inputdelayed dynamics," IEEE Control Systems Letters, vol. 1, pp. 116-121, July 2017. 
23. W. Respondek and I. Tall, Feedback Equivalence of Nonlinear Control Systems: A Survey on Formal Approaches, pp. 137-262. 10.1201/9781420027853.ch4, CRC Press, 2016/10/18 2005.

24. C. Califano, L. Marquez-Martinez, and C. Moog, "Linearization of time-delay systems by input-output injection and output transformation," Automatica, vol. 49, no. 6, pp. 1932-1940, 2013.

25. P. Di Giamberardino, S. Monaco, and D. Normand-Cyrot, "On equivalence and feedback equivalence to finitely computable sampled models," in Proceedings of the 45th IEEE Conference on Decision and Control, pp. 5869-5874, 10.1109/CDC.2006.377699, 2006.

26. P. Di Giamberardino, S. Monaco, and D. Normand-Cyrot, "Why multirate sampling is instrumental for control design purpose: the example of the one-leg hopping robot," in Proceedings of the 41st IEEE CDC, vol. 3, pp. 3249 - 3254, 2002.

27. S. Monaco, D. Normand-Cyrot, and F. Tiefensee, "Sampled-data stabilization; a pbc approach," IEEE Trans. on Automatic Control, vol. 56, pp. 907-912, 2011.

28. V. Tanasa, S. Monaco, and D. Normand-Cyrot, "Backstepping control under multi-rate sampling," IEEE Transactions on Automatic Control, vol. 61, no. 5, pp. 1208 - 1222, 2016.

29. A. Astolfi and R. Ortega, "Immersion and invariance: a new tool for stabilization and adaptive control of nonlinear systems," IEEE Transactions on Automatic Control, vol. 48, no. 4, pp. 590-606, 2003.

30. A. Astolfi, D. Karagiannis, and R. Ortega, Nonlinear and adaptive control with applications. Springer Publishing Company, 2008.

31. Y. Yalcin and A. Astolfi, "Immersion and invariance adaptive control for discrete time systems in strict feedback form," Systems \& Control Letters, vol. 61, no. 12, pp. 1132 - 1137, 2012.

32. M. Mattioni, S. Monaco, and D. Normand-Cyrot, "Digital stabilization of strict feedback dynamics through immersion and invariance," in Proc. IFAC MICNON, (St Petersbourg), pp. 1085-1090, 2015.

33. M. Mattioni, S. Monaco, and D. Normand-Cyrot, "Immersion and invariance stabilization of strict-feedback dynamics under sampling," Automatica, vol. 76, pp. 78 - 86, 2017. 